\title{
Tension-type headache associated with obstructive sleep apnea: a nationwide population-based study
}

\author{
Yu-Chwen Chiu' ${ }^{1}$ Hsiao-Yun Hü ${ }^{2,3}$, Fei-Peng Lee ${ }^{4,5}$ and Hung-Meng Huang ${ }^{1,4^{*}}$
}

\begin{abstract}
Background: There is still controversy regarding the association between primary headaches and obstructive sleep apnea. We explored the relationship between tension-type headache (TTH) and obstructive sleep apnea (OSA) using a large nationwide population-based data set in Taiwan.

Methods: We identified 4759 patients diagnosed with OSA from the Taiwan Longitudinal Health Insurance Database, based on polysomnography, as the OSA group. We then randomly selected 19036 subjects without OSA, matched by sex and age, to serve as the non-OSA group. The multivariate Cox proportional hazards model with matching for age and sex was used to assess the possible associations between TTH and OSA among the patients.

Results: The prevalence of TTH was $10.2 \%$ among OSA patients and $7.7 \%$ among non-OSA patients $(p<0.001)$. The multivariate Cox proportional hazards model revealed patients with OSA were more likely to have TTH (hazard ratio, $1.18 ; 95 \% \mathrm{Cl}, 1.06-1.31)(p=0.003)$ than patients in the non-OSA group.

Conclusion: Patients with OSA had a higher likelihood of developing TTH than patients in the non-OSA group. Further studies of physiological patterns between OSA and TTH are needed to confirm the study findings.

Keywords: Polysomnography; Sleep study; Health insurance database; Taiwan; Sleep disturbance; Headache; Hypopnea
\end{abstract}

\section{Background}

Obstructive sleep apnea (OSA) is by far the most common sleep disturbance, affecting some $24 \%$ of men and $9 \%$ of women by the time they reach middle age [1]. Repeated episodes of apnea or hypopnea caused by upper airway blockage during sleep characterize OSA. A typical measurement of sleep apnea is the apnea-hypopnea index. This is an average that represents the combined number of episodes of apnea and hypopnea that occur per hour of sleep, which is calculated from the results of overnight polysomnography. The definitive diagnostic test for OSA is polysomnography. Obstructive sleep apnea is receiving increased attention as an important cause of medical morbidity, including excessive daytime

\footnotetext{
* Correspondence: hmengh@yahoo.com.tw

'Department of Otolaryngology, Taipei City Hospital, Taipei, Taiwan ${ }^{4}$ Department of Otolaryngology, School of Medicine, College of Medicine, Taipei Medical University, Taipei, Taiwan

Full list of author information is available at the end of the article
}

sleepiness, metabolic derangement, and cardiovascular disease.

People in the general population frequently experience headache pain, placing a burden on health care and other economic expenditures. Headaches may be intrinsically related to sleep, may cause sleep disturbances, or manifestations of sleep apnea. The International Classification of Headache Disorders (ICHD II) [2] includes two specific diagnoses for sleep-related headaches, "sleep apnea headache" and "hypnic headache". Sleep apnea headaches present upon awakening with an apneahypopnea index $\geq 5$, as demonstrated by overnight PSG. Hypnic headaches develop only during sleep and always awaken patients. Nevertheless, sleep disorders may still be associated with other primary headaches, especially tension-type headaches (TTH).

Several studies [3-6] have illustrated positive correlations between TTH and sleep disturbances including sleep apnea. Nevertheless, several studies [7-9] have shown no 
correlation. In a recent cross-sectional population-based study, Kristiansen et al. [8,9] showed that sleep apnea had little if any influence on the development of TTH. Therefore, the goal of this research was to explore the possible association between TTH and OSA within a nationwide, population-based data set in Taiwan.

\section{Methods}

\section{Data sources}

Taiwan's National Health Insurance (NHI) program, which started March 1, 1995, provides comprehensive coverage for medical care of people living in Taiwan. This program was built on the concept of mutual assistance, and it depends on the insured paying their premiums according to regulations. At the end of 2012, there were 23 million people (approximately $99 \%$ of Taiwan's population) enrolled in the program. The Bureau of NHI collects data from the NHI program and sends it to the National Health Research Institutes (NHRI) to form the original files of National Health Insurance Research Database (NHIRD). The study used data from the Longitudinal Health Insurance Database 2000 (LHID2000), which was created by the NHRI and contained all the original claims data for one million individuals (approximately 5\% of Taiwan's population) randomly sampled from the 2000 Registry of Beneficiaries of the NHIRD. The NHRI confirmed that there was no significant difference in gender distribution between the patients in the LHID2000 and the original NHIRD.

All information allowing a specific patient to be identified was encrypted. The encryption procedure was consistent between datasets; thus, all claims belonging to each patient were linked. The confidentiality of the data abides by the data regulations of the Bureau of National Health Insurance. The Institutional Review Board of Taipei City Hospital approved this study (IRB no.: TCHIRB-1020715-W).

\section{Study sample}

We first identified all patients with a new diagnosis of OSA (International Classification of Diseases, Ninth Revision, Clinical Modification [ICD-9-CM] codes 327.23, $780.51,780.53$, or 780.57 ) based on polysomnography $(\mathrm{AHI} \geq 5)$ between January 1, 2004 and December 31, $2010(\mathrm{n}=5023)$. We excluded patients younger than 18 years from the analysis $(n=264)$. For each case, the date of the first diagnosis was assigned as the index date. Finally, the OSA group included 4759 patients.

We selected 19,036 patients without OSA (four for each OSA case) from the remaining subjects of the LHID2000 as the non-OSA group. The patients in the non-OSA group were matched with the case subjects in OSA group by sex, age group $(<30,30-39,40-49,50-59, \geq 60)$, and year of the index date. None of the selected non-OSA patients had been diagnosed with OSA after 1995, the initiation year of the NHI program.

We also investigated the pre-existing comorbidities of hypertension (ICD-9-CM 401), epilepsy (ICD-9-CM 345), major depression disorders (ICD-9-CM 296.2 and 296.3), and anxiety disorders (ICD-9-CM 300.00, 300.01, 300.02 and 300.09). We selected these comorbidities due to their possible associations with OSA and TTH as documented in earlier studies [10-14].

Cases of TTH were identified based on their corresponding ICD-9-CM codes. We used ICD-9-CM codes 307.81 and 339.1 for TTH. Neurologists diagnosed all headaches. We included patients in this group only when TTH was diagnosed after the OSA index date.

\section{Statistical analysis}

SAS version 9.3 (SAS Institute Inc., Cary, NC, USA) was used to link the data in the LHID2000; Stata 13 (Stata Corporation, College Station, TX, USA) was used to perform the statistical analyses. Demographic information of patients with or without OSA were compared using a $\chi 2$ test for categorical variables. The threshold for statistical significance was set at two-tailed $\mathrm{P}$ value $<0.05$. The risk of TTH among OSA patients and OSA-free group between January 1, 2004 and December 31, 2010 was analyzed. The $\log -\log$ survival curve was used to test the proportional assumption. The log-log plots indicated that the data might be suitable for the Weibull distribution parameters estimation. A sensitivity test of the Weibull model was performed. Cox proportional hazards regression model was used to estimate the adjusted hazard ratio (HR) and 95\% confidence intervals (CI) for developing TTH. The analysis included potential correlation factors of TTH such as hypertension, epilepsy, major depression disorder, and anxiety disorder. Finally, HRs were calculated for specific subgroups with adequately adjusted regression models.

\section{Results}

After matching for sex and age, we found that patients with OSA had higher proportions of certain comorbidities than the non-OSA group did (Table 1), including hypertension ( $38.3 \%$ vs. $30.2 \%, p<0.001)$, epilepsy $(2.5 \%$ vs. $1.6 \%, p<0.001)$, major depression disorder $(5.5 \%$ vs. $2.7 \%, p<0.001)$, and anxiety disorder $(26.7 \%$ vs. $15.3 \%$, $p<0.001$ ). Table 1 shows the relationships between sociodemographic characteristics and OSA. Patients with OSA were more likely to have monthly incomes of NT\$ 40,000 or higher $(p<0.001)$ and live in northern and central Taiwan $(p<0.001)$.

The prevalence of TTH was $10.2 \%$ among OSA patients, and was higher than in non-OSA patients $(7.7 \%$, $p<0.001$ ).

Table 2 shows the incidence rates (IR) in each group over the 6-year follow-up period. The incidence rate of 
Table 1 Demographic characteristics of patients with obstructive sleep apnea (OSA) and non-OSA-diagnosis patients from 2004 to 2010

\begin{tabular}{|c|c|c|c|c|c|}
\hline \multirow[t]{2}{*}{ Variable } & \multicolumn{2}{|c|}{ Matched controls } & \multicolumn{2}{|c|}{ Patients with OSA } & \multirow[t]{2}{*}{$p$-value } \\
\hline & $(\mathrm{N}=19036)$ & $\%$ & $(\mathrm{~N}=4759)$ & $\%$ & \\
\hline Sex & & & & & 1.000 \\
\hline Female & 5,524 & 29.0 & 1,381 & 29.0 & \\
\hline Male & 13,512 & 71.0 & 3,378 & 71.0 & \\
\hline Age & & & & & 1.000 \\
\hline$<30$ & 1,956 & 10.3 & 489 & 10.3 & \\
\hline $30-39$ & 3,780 & 19.9 & 945 & 19.9 & \\
\hline $40-49$ & 4,720 & 24.8 & 1,180 & 24.8 & \\
\hline $50-59$ & 4,768 & 25.0 & 1,192 & 25.0 & \\
\hline$\geq 60$ & 3,812 & 20.0 & 953 & 20.0 & \\
\hline Monthly Income, NT\$ & & & & & $<0.001$ \\
\hline$<20,000$ & 2,285 & 12.0 & 515 & 10.8 & \\
\hline $20,000-39,999$ & 6,599 & 34.7 & 1,461 & 30.7 & \\
\hline$\geq 40,000$ & 4,447 & 23.4 & 1,588 & 33.4 & \\
\hline Union/association member & 2,647 & 13.9 & 455 & 9.6 & \\
\hline Local government enrollee & 3,058 & 16.1 & 740 & 15.5 & \\
\hline Region & & & & & $<0.001$ \\
\hline Northern & 8,529 & 44.8 & 2,476 & 52.0 & \\
\hline Central & 4,131 & 21.7 & 1,434 & 30.1 & \\
\hline Southern & 5,943 & 31.2 & 811 & 17.0 & \\
\hline Eastern & 433 & 2.3 & 38 & 0.8 & \\
\hline \multicolumn{6}{|l|}{ Comorbidity } \\
\hline Hypertension & 5,755 & 30.2 & 1,823 & 38.3 & $<0.001$ \\
\hline Epilepsy & 297 & 1.6 & 121 & 2.5 & $<0.001$ \\
\hline Major depression disorder & 520 & 2.7 & 264 & 5.5 & $<0.001$ \\
\hline Anxiety disorder & 2,911 & 15.3 & 1,270 & 26.7 & $<0.001$ \\
\hline Tension type headache & & & & & $<0.001$ \\
\hline No & 17,567 & 92.3 & 4,275 & 89.8 & \\
\hline Yes & 1,469 & 7.7 & 484 & 10.2 & \\
\hline Total & 19,036 & & 4,759 & & \\
\hline
\end{tabular}

TTH was higher in OSA patients (37 per 1000 personyears) than in non-OSA patients (28 per 1000 personyears).

We used multivariate Cox proportional hazards regression model for predicting the chances of developing TTH (Table 3). After adjustment for sex, age, sociodemographic factors, and comorbidities, patients with OSA were more likely to have TTH $(\mathrm{HR}=1.18,95 \% \mathrm{CI}$ $1.06-1.31, p=0.003)$ than patients in the non-OSA group. Patients with hypertension tended to have TTH $(\mathrm{HR}=1.28,95 \%$ CI $1.16-1.42, p<0.001)$.

Among affective disorders, patients with major depression disorder were more likely to have TTH $(\mathrm{HR}=1.73$, 95\% CI 1.47-2.03, $p<0.001)$. Patients with anxiety disorder also had greater chances of developing TTH $(\mathrm{HR}=2.30,95 \%$ CI $2.09-2.54, p<0.001)$.

We found that men had a lower chance of having TTH $(\mathrm{HR}=0.61,95 \%$ CI $0.56-0.67, p<0.001)$ than did women. TTH occurred more often among older age adults (age $50-59$ years, HR $=1.76,95 \%$ CI $1.42-2.18$, $p<0.001)$.

Regarding economic condition and geographic location, we found that the chances of developing TTH increased when monthly income was less than NT\$ 20,000 and patients resided outside of northern Taiwan.

Table 4 shows the subgroup analysis of the variables. Regardless of gender, patients with OSA were more likely to have TTH than were non-OSA patients. Patients with 
Table 2 The incidences of tension-type headache in each group from 2004 to 2010

\begin{tabular}{|c|c|c|c|}
\hline \multirow[t]{2}{*}{ Variables } & \multicolumn{3}{|c|}{ Tension-type headache } \\
\hline & Person-Years & TTH (N) & IR (\%o/yr) \\
\hline Total & 64,864 & 1,953 & 30 \\
\hline \multicolumn{4}{|l|}{ Sex } \\
\hline Female & 18,102 & 810 & 45 \\
\hline Male & 46,762 & 1,143 & 24 \\
\hline \multicolumn{4}{|l|}{ Age } \\
\hline$<30$ & 6,938 & 104 & 15 \\
\hline $30-39$ & 13,122 & 316 & 24 \\
\hline $40-49$ & 16,460 & 464 & 28 \\
\hline $50-59$ & 15,996 & 579 & 36 \\
\hline$\geq 60$ & 12,348 & 490 & 40 \\
\hline \multicolumn{4}{|l|}{ Monthly Income } \\
\hline$<20,000$ & 9,122 & 217 & 24 \\
\hline $20,000-39,999$ & 20,610 & 628 & 30 \\
\hline$\geq 40,000$ & 16,365 & 384 & 23 \\
\hline Union/association member & 8,564 & 363 & 42 \\
\hline Local government enrollee & 10,202 & 361 & 35 \\
\hline \multicolumn{4}{|l|}{ Region } \\
\hline Northern & 30,023 & 749 & 25 \\
\hline Central & 14,865 & 448 & 30 \\
\hline Southern & 18,709 & 719 & 38 \\
\hline Eastern & 1,266 & 37 & 29 \\
\hline \multicolumn{4}{|l|}{ Comorbidy } \\
\hline Hypertension & 21,434 & 882 & 41 \\
\hline Epilepsy & 1,153 & 34 & 29 \\
\hline Major drpression disorder & 2,226 & 177 & 80 \\
\hline Anxiety disorder & 11,579 & 769 & 66 \\
\hline \multicolumn{4}{|l|}{ Obstructive sleep apnea } \\
\hline No & 51,824 & 1,469 & 28 \\
\hline Yes & 13,040 & 484 & 37 \\
\hline
\end{tabular}

$p$-value $<0.001$ in all results.

IR: incidence rate.

OSA 50-59 years of age with monthly income of NT\$ 40,000 or more had a statistically significant association with TTH. All OSA patients, even those without the selected comorbidities, showed a statistically significant association with TTH compared to non-OSA patients.

\section{Discussion}

According to our results, obstructive sleep apnea is associated with gender, age, sociodemographic characteristics, metabolic consequences, and affective disorders (Table 1). TTH is the most common primary headache. Nonetheless, there has been much less study of TTH compared with migraine. It is likely that patients suffer
Table 3 Multivariate Cox regression model for predictor of tension-type headache development

\begin{tabular}{|c|c|c|c|}
\hline \multirow[t]{2}{*}{ Variable } & \multicolumn{3}{|c|}{ Tension-type headache } \\
\hline & HR & $95 \% \mathrm{Cl}$ & $p$-value \\
\hline \multicolumn{4}{|l|}{ Sex } \\
\hline Female & 1.00 & & \\
\hline Male & 0.61 & $0.56-0.67$ & $<0.001$ \\
\hline \multicolumn{4}{|l|}{ Age } \\
\hline$<30$ & 1.00 & & \\
\hline $30-39$ & 1.56 & $1.25-1.94$ & $<0.001$ \\
\hline $40-49$ & 1.58 & $1.27-1.96$ & $<0.001$ \\
\hline $50-59$ & 1.76 & $1.42-2.18$ & $<0.001$ \\
\hline$\geq 60$ & 1.65 & $1.31-2.06$ & $<0.001$ \\
\hline \multicolumn{4}{|l|}{ Monthly Income } \\
\hline$<20,000$ & 1.00 & & \\
\hline $20,000-39,999$ & 1.34 & $1.14-1.56$ & $<0.001$ \\
\hline$\geq 40,000$ & 1.09 & $0.92-1.30$ & 0.298 \\
\hline Union/association member & 1.51 & $1.27-1.80$ & $<0.001$ \\
\hline Local government enrollee & 1.35 & $1.14-1.60$ & $<0.001$ \\
\hline \multicolumn{4}{|l|}{ Region } \\
\hline Northern & 1.00 & & \\
\hline Central & 1.09 & $0.96-1.23$ & 0.173 \\
\hline Southern & 1.50 & $1.35-1.67$ & $<0.001$ \\
\hline Eastern & 1.06 & $0.76-1.48$ & 0.720 \\
\hline \multicolumn{4}{|l|}{ Comorbidy } \\
\hline Hypertension & 1.28 & $1.16-1.42$ & $<0.001$ \\
\hline Epilepsy & 0.79 & $0.56-1.11$ & 0.170 \\
\hline Major drpression disorder & 1.73 & $1.47-2.03$ & $<0.001$ \\
\hline Anxiety disorder & 2.30 & $2.09-2.54$ & $<0.001$ \\
\hline \multicolumn{4}{|l|}{ Obstructive sleep apnea } \\
\hline No & 1.00 & & \\
\hline Yes & 1.18 & $1.06-1.31$ & 0.003 \\
\hline
\end{tabular}

less with TTH than other chronic headaches. A recent study showed that headache patients who do not seek medical assistance are more likely to have TTH [15]. TTH is associated with certain mental illnesses, especially anxiety and major depression disorder [16]. We extracted possible overlapping variables from the database to make clear their direct or indirect effects on migraine and TTH.

In accordance with our findings, one cross-sectional population based study with 297 participants enrolled from Norway used a modified short form of the Karolinska Sleep Questionnaire and Epworth Sleepiness Scale. They found severe sleep disturbance was three times more likely for individuals with TTH [6]. Authors of another retrospective analysis reported that among 82 chronic headache patients with migraine or TTH or both, 52 
Table 4 Risk comparison of tension-type headache between OSA and non-OSA participants

\begin{tabular}{|c|c|c|c|}
\hline \multirow[t]{2}{*}{ Variable } & \multirow[t]{2}{*}{$\mathbf{N}$} & \multicolumn{2}{|c|}{ Tension-type headache } \\
\hline & & HR & $95 \% \mathrm{Cl}$ \\
\hline \multicolumn{4}{|l|}{ Sex } \\
\hline Female & 6,905 & 1.17 & $1.00-1.39$ \\
\hline Male & 16,890 & 1.17 & $1.02-1.35$ \\
\hline \multicolumn{4}{|l|}{ Age } \\
\hline$<30$ & 2,445 & 1.27 & $0.80-2.00$ \\
\hline $30-39$ & 4,725 & 1.03 & $0.78-1.35$ \\
\hline $40-49$ & 5,900 & 1.40 & $1.13-1.73$ \\
\hline $50-59$ & 5,960 & 1.29 & $1.06-1.56$ \\
\hline$\geq 60$ & 4,765 & 0.93 & $0.74-1.17$ \\
\hline \multicolumn{4}{|l|}{ Monthly Income } \\
\hline$<20,000$ & 2,800 & 1.05 & $0.75-1.47$ \\
\hline $20,000-39,999$ & 8,060 & 1.16 & $0.96-1.41$ \\
\hline$\geq 40,000$ & 6,035 & 1.37 & $1.11-1.70$ \\
\hline Union/association member & 3,102 & 0.88 & $0.65-1.18$ \\
\hline Local government enrollee & 3,798 & 1.22 & $0.96-1.54$ \\
\hline \multicolumn{4}{|l|}{ Region } \\
\hline Northern & 11,005 & 1.22 & $1.04-1.43$ \\
\hline Central & 5,565 & 1.25 & $1.02-1.53$ \\
\hline Southern & 6,754 & 0.99 & $0.80-1.23$ \\
\hline Eastern & 471 & 2.47 & $0.90-6.81$ \\
\hline \multicolumn{4}{|l|}{ Comorbidity } \\
\hline \multicolumn{4}{|l|}{ Hypertension } \\
\hline No & 16,217 & 1.22 & $1.06-1.42$ \\
\hline Yes & 7,578 & 1.08 & $0.92-1.26$ \\
\hline \multicolumn{4}{|l|}{ Epilepsy } \\
\hline No & 23,377 & 1.19 & $1.07-1.32$ \\
\hline Yes & 418 & 0.75 & $0.31-1.82$ \\
\hline \multicolumn{4}{|l|}{ Major depression disorder } \\
\hline No & 23,011 & 1.18 & $1.06-1.32$ \\
\hline Yes & 784 & 1.18 & $0.86-1.61$ \\
\hline \multicolumn{4}{|l|}{ Anxiety disorder } \\
\hline No & 19,614 & 1.25 & $1.08-1.45$ \\
\hline Yes & 4,181 & 1.10 & $0.94-1.28$ \\
\hline
\end{tabular}

Each factor was adjusted for all other factors.

patients (63\%) also had OSA. The headache improvement rate was up to $49 \%$ after receiving either standard medical therapy or continuous positive airway pressure therapy [17].

The main finding of our study showed a positive relationship between OSA and TTH. Regardless of gender, patients with OSA were more likely to have TTH after adjustment for age, residential region, monthly income, and possible comorbidities. In Table 4, patients with OSA showed stronger positive associations with TTH in most comorbidity-free groups than in comorbidity groups. We assumed patients with the selected comorbidities had poorer health-related quality of life and did not undergo OSA testing, which is not considered an emergency, but needs to be confirmed by a time-consuming polysomnography study.

Lack of sleep itself is related to headaches. Common sleep disorders include OSA, central sleep apnea, insomnia, periodic limb movement disorder [18], and bruxism [19]. The mechanisms by which headaches are associated with specific sleep disturbances are complex and manifold. In cluster headaches, oxygen desaturation below $89 \%$ during sleep preceded 8 out of 14 attacks of cluster headache among 10 patients [20], giving positive support for the relationship of cluster headache and OSA.

Serotonin and several other neurotransmitters influence TTH. Pain thresholds elicited in patients with chronic, painful pathologies are linked to serum serotonin levels [21]. Additionally, many TTH patients show changes in serotonin levels [22-26]. Interestingly, serotonin dysfunction may also play a role in OSA $[27,28]$. Serotonin delivery is reduced to upper airway dilator motor neurons during sleep, and this contributes to sleep-related reductions in dilator muscle activity and thus, upper airway obstruction [27]. Fluctuation of serotonin concentrations could be a primary or secondary factor common to different mechanisms. Such a hypothesis provides a possible explanation for the manifestation of OSA and TTH. Furthermore, a growing number of studies show that sleep disturbance increases pain sensitivity and exacerbates chronic pain, including fibromyalgia, rheumatoid arthritis, and headache [29-31]. Smith et al. [32] suggested that sleep fragmentation impairs endogenous pain inhibitory function and increases spontaneous pain in middle-aged healthy women. Lentz et al. [33] found that slow wave sleep disruption without reducing total sleep was also associated with decreased pain thresholds in middle-aged women. Khalid et al. [34] demonstrated that patients with severe OSA were hyperalgesic, and their pain sensitivity improved with continuous positive airway pressure treatment, and the improvement disappeared immediately with discontinuation of continuous positive airway pressure therapy. We suggest patients with refractory TTH undergo evaluation for OSA. Treating OSA might not only improve headaches, but also decrease the risk of related comorbidities.

\section{Clinical importance and limitations}

In this study, we utilized a nationwide population database to investigate the epidemiologic associations between OSA and TTH. One of the key strengths of the study, in addition to a large sample size, was selecting patients with an OSA diagnosis confirmed by polysomnography. We 
believe this selection criterion is more precise and strict than patient recall in response to a questionnaire. Nonetheless, this could also lead to underestimation of the enrolled OSA patients in the total population. The National Health Insurance Administration in Taiwan samples the medical charts routinely and makes detailed assessments for accuracy of medical coding. The reliability and validity of the NHI research database for epidemiologic investigation has been reported [35].

A key limitation of the study is the relatively low prevalence of OSA and headache. The prevalence of TTH in the general population varies from $30 \%$ to $78 \%$ (2) because of differences in research study design. A diagnosis of TTH may be overlooked or delayed because symptoms can be mild or infrequent. In addition, some symptoms can mimic TTH of other conditions, such as acute or chronic paranasal sinusitis, transient ischemic attack, meningitis, chronic subdural hemorrhage, and brain tumor. TTH diagnoses of patients enrolled in our study were made by neurologists, which means we may have reliable accuracy of the clinical diagnoses in our study. Similarly, according to epidemiological studies, the prevalence rates of OSA in the general population vary from $3 \%$ to $7 \%$ [36]. It was about $0.48 \%$ (4759/ 1000000) in our study due to the strict selection of polysomnography-confirmed cases only. A disadvantage of our study is that we could not have face-to-face interviews with patients. Thus, we can only set higher limitations on case selection to lower inaccuracy from such a large study sample. Even if the prevalence were underestimated, we still would have convincing findings.

Another limitation of the study is that the degree of OSA and the pattern of headache attack could not be determined from the database. Thus, we could not determine whether the severity of OSA influenced TTH frequency, duration, and pain characteristics. We could not differentiate TTH from episodic and chronic type headaches. Additionally, some possibly relevant confounding factors, such as body mass index, smoking, and alcohol consumption were not included in the data set. Therefore, they were not analyzed.

In our findings, the incidence rates (Table 2) over the 6year follow-up period (from 2004 to 2010, 30 to 78 per 1,000 person-years) were higher than some earlier studies (13). Not being able to completely exclude recurrences should be taken into account, especially in older age group. The substantially increasing trend in the incidence of frequent headache in past 10 years was also found in other studies [37]. Additional researches on the occurrence and causes of increasing headache incidences are needed.

\section{Conclusions}

Our results support the notion that patients with OSA are more likely to develop TTH. The mechanism underlying this association is not clear but includes changes in serotonin levels. Further studies of physiological patterns of OSA and TTH are needed to confirm the study findings and to enrich our understanding of their interactions.

\section{Abbreviations \\ TTH: Tension-type headache; OSA: Obstructive sleep apnea; NHRI: National health research institutes; NHIRD: National health insurance research database; LHID2000: Longitudinal health insurance database 2000; ICD-9- CM: International classification of diseases, ninth revision, clinical modification; HR: Hazard ratio; Cl: Confidence interval.}

\section{Competing interests}

The authors declare that they have no competing interests.

\section{Authors' contributions}

Chiu YC and Huang HM prepared the study design and initial draft. Hu HY performed the statistical analysis. Lee FP had the original idea of the study. All authors have contributed to the practical plans for the study, revised and approved the final manuscript.

\section{Acknowledgements}

This work was supported by a grant from Taipei City Hospital (grant no.: TPECH-103-06).

\section{Author details}

${ }^{1}$ Department of Otolaryngology, Taipei City Hospital, Taipei, Taiwan. ${ }^{2}$ Department of Education and Research, Taipei City Hospital, Taipei, Taiwan. ${ }^{3}$ Department of Public Health, Institute of Public Health, National Yang Ming University, Taipei, Taiwan. ${ }^{4}$ Department of Otolaryngology, School of Medicine, College of Medicine, Taipei Medical University, Taipei, Taiwan. ${ }^{5}$ Department of Otolaryngology, Wan Fang Hospital, Taipei Medical University, Taipei, Taiwan.

Received: 8 March 2015 Accepted: 11 April 2015

Published online: 21 April 2015

\section{References}

1. Young T, Palta M, Dempsey J, Skatrud J, Weber S, Badr S (1993) The occurrence of sleep-disordered breathing among middle-aged adults. N Engl J Med 328:1230-5

2. Headache Classification Subcommittee of the International Headache Society (2004) The international classification of headache disorders, 2nd Edition. Cephalalgia 24:1-160

3. Mitsikostas DD, Vikelis M, Viskos A (2008) Refractory chronic headache associated with obstructive sleep apnoea syndrome. Cephalalgia 28(2):139-43

4. Karthik N, Sinha S, Taly AB, Kulkarni GB, Ramachandraiah CT, Rao S (2013) Alternation in polysomnographic profile in 'migraine without aura' compared to healthy controls. Sleep Med 14(2):211-4

5. Yeung WF, Chung KF, Wong CY (2010) Relationship between insomnia and headache in community-based middle-aged Hong Kong Chinese women. J Headache Pain 11(3):187-95

6. Odegard SS, Engstrom M, Sand T, Stovner LJ, Zwart JA, Hagen K (2010) Associations between sleep disturbance and primary headaches: the third Nord-Trondelag Health Study. J Headache Pain 11(3):197-206

7. Jensen R, Olsborg C, Salvesen R, Torberqsen T, Bekkelund SI (2004) Is obstructive sleep apnea syndrome associated with headache? Acta Neurol Scand 109(3):180-4

8. Kristiansen HA, Kværner KJ, Akre H, Overland B, Russell MB (2011) Tension-type headache and sleep apnea in the general population. J Headache Pain 12(1):63-9

9. Kristiansen HA, Kværner KJ, Akre H, Overland B, Russell MB (2011) Migraine and sleep apnea in the general population. J Headache Pain 12(1):55-61

10. Huang QR, Qin Z, Zhang S, Chow CM (2008) Clinical patterns of obstructive sleep apnea and its comorbid conditions: a data mining approach. J Clin Sleep Med 4(6):543-50

11. Drager LF, Togeiro SM, Polotsky VY, Lorenzi-Filho G (2013) Obstructive sleep apnea: a cardiometabolic risk in obesity and the metabolic syndrome. J Am Coll Cardiol 62(7):569-76 
12. Babson KA, Del Re AC, Bonn-Miller MO, Woodward SH (2013) The comorbidity of sleep apnea and mood, anxiety, and substance use disorders among obese military veterans within the Veterans Health Administration. J Clin Sleep Med 9(12):1253-8

13. Jensen R, Stovner $L J$ (2008) Epidemiology and comorbidity of headache. Lancet Neurol 7(4):354-61

14. Scher Al, Bigal ME, Lipton RB (2005) Comorbidity of migraine. Curr Opin Neurol 18(3):305-10

15. Oliveira DR, Leite AA, Rocha-Filho PA (2011) Which patients with headache do not seek medical attention. Headache 51(8):1279-84

16. Juang KD, Wang SJ, Fuh JL, Lu SR, Su TP (2000) Comorbidity of depressive and anxiety disorders in chronic daily headache and its subtypes. Headache 40(10):818-23

17. Johnson KG, Ziemba AM, Garb JL (2013) Improvement in headaches with continuous positive airway pressure for obstructive sleep apnea:a retrospective analysis. Headache 53(2):333-43

18. Esposito M, Parisi P, Miano S, Carotenuto M (2013) Migraine and periodic limb movement disorders in sleep in children:a preliminary case-control study. J Headache Pain 14:57

19. Bailey DR (1990) Tension headache and bruxism in the sleep disordered patient. J Craniomandib Pract 8:174-82

20. Kudrow L, MacGinty DJ, Phillips ER, Stevenson M (1984) Sleep apnea in cluster headache. Cephalalgia 4(1):33-8

21. Jung AC, Staiger T, Sullivan M (1997) The efficacy of selective serotonin reuptake inhibitors for the management of chronic pain. J Gen Intern Med 12:384-9

22. Sicuteri F (1972) Headache as possible expression of deficiency of brain 5hydroxytryptamine (central denervation supersensitivity). Headache 12:69-72

23. Ferrari MD, Odink J, Tapparelli C, Van Kempen GM, Pennings EJ, Bruyn GW (1989) Serotonin metabolism in migraine. Neurology 39(9):1239-42

24. Lothe A, Merlet I, Demarquay G, Costes N, Ryvlin P, Mauguière F (2008) Interictal brain 5-HT1A receptors binding in migraine without aura: an 18 F-MPPF-PET study. Cephalalgia 28(12):1282-91

25. Aylin A, Cenqiz T, Emin E, Neriman A, Sacide P (2008) Serotonin transporter gene polymorphisms in patients with chronic tension-type headache: a preliminary study. Neurol India 56(2):156-60

26. Bendtsen L (2000) Central sensitization in tension-type headache-possible pathophysiological mechanisms. Cephalalgia 20:486-508

27. Veasey SC (2003) Serotonin agonists and antagonists in obstructive sleep apnea: therapeutic potential. Am J Respir Med 2:21-9

28. McCann UD, Sgambati FP, Schwartz AR, Ricaurte GA (2009) Sleep apnea in young abstinent recreational MDMA ("ecstasy") consumers. Neurology 73(23):2011-7

29. Lautenbacher S, Rollman GB (1997) Possible deficiencies of pain modulation in fibromyalgia. Clin J Pain 13:189-96

30. Pielsticker A, Haag G, Zaudig M, Lautenbacher S (2005) Impairment of pain inhibition in chronic tension-type headache. Pain 118(1-2):215-23

31. Leffler AS, Kosek E, Lerndal T, Nordmark B, Hansson P (2002) Somatosensory perception and function of diffuse noxious inhibitory controls (DNIC) in patients suffering from rheumatoid arthritis. Eur J Pain 6(2):161-76

32. Smith M, Edwards R, McCann U, Haythornthwaite JA (2007) The effects of sleep deprivation on pain inhibition and spontaneous pain in women. SLEEP 30(4):494-505

33. Lentz MJ, Landis CA, Rothermel J, Shaver JL (1999) Effects of selective slow wave sleep disruption on musculoskeletal pain and fatigue in middle aged women. J Rheumatol 26(7):1586-92

34. Khalid I, Roehrs TA, Hudgel DW, Roth T (2011) Continuous positive airway pressure in severe obstructive sleep apnea reduces pain sensitivity. SLEEP 34(12):1687-91

35. Cheng CL, Kao YH, Lin SJ, Lee CH, Lai ML (2011) Validation of the national health insurance research database with ischemic stroke cases in Taiwan. Pharmacoepidemiol Drug Saf 20(3):236-42

36. Punjabi NM (2008) The epidemiology of adult obstructive sleep apnea. Proc Am Thorac Soc 5:136-43

37. Anttila P, Metsähonkala L, Sillanpää M (2006) Long-term trends in the incidence of headache in Finnish schoolchildren. Pediatrics 117:1197-201

\section{Submit your manuscript to a SpringerOpen ${ }^{\circ}$ journal and benefit from:}

- Convenient online submission

Rigorous peer review

- Immediate publication on acceptance

- Open access: articles freely available online

- High visibility within the field

- Retaining the copyright to your article

Submit your next manuscript at $>$ springeropen.com 